\title{
Prophylactic effect of citalopram in unipolar, recurrent depression
}

\author{
Placebo-controlled study of maintenance therapy ${ }^{\dagger}$ \\ B. HOCHSTR ASSER, P. M. ISAKSEN, H. KOPONEN, L. LAURITZEN, \\ F. A. MAHNERT, F. ROUILLON, A. G. WADE, M. ANDERSEN, S. F. PEDERSEN, \\ J. C. G. DE SWART and R. NIL
}

\author{
Background Major depression is \\ highly recurrent. Antidepressant \\ maintenance treatment has proven \\ efficacy against recurrent depression.
}

\begin{abstract}
Aims Comparison of prophylactic efficacy of citalopram versus placebo in unipolar, recurrent depression.
\end{abstract}

Methods Patients 18-65 years of age with recurrent unipolar major depression (DSM-IV), a Montgomery-Åsberg Depression Rating Scale score of $\geqslant 22$ and two or more previous depressive episodes, one within the past 5 years, were treated openly with citalopram (20-60 mg) for 6-9 weeks and, if responding, continued for 16 weeks before being randomised to double-blind maintenance treatment with citalopram or placebo for 48-77 weeks.

Results A total of 427 patients entered acute treatment and 269 were randomised to double-blind treatment. Time to recurrence was longer in patients taking citalopram than in patients taking placebo $(P<0.001)$. Prophylactic treatment was well tolerated.

Conclusions Citalopram (20,40 and $60 \mathrm{mg}$ ) is effective in the prevention of depressive recurrences. Patients at risk should continue maintenance treatment at the dose necessary to resolve symptoms in the acute treatment phase.

\section{Declaration of interest Funded by} H. Lundbeck A/S.

†See editorial, pp. 294-295, this issue.
Major depression has a life-time prevalence of $17 \%$, is highly recurrent and is often lifelong (Kessler et al, 1994). Prospective studies of unipolar depression revealed recurrence rates of up to $85 \%$, with an average episode duration of about 6 months (Angst, 1980; Mueller et al, 1999). Recurrence risk increases with duration and number of episodes. To address the episodic nature of depression, Frank et al (1991) conceptualised three phases of antidepressant treatment: acute treatment (resolution of depressive symptoms), continuation treatment (4-6 months' relapse prevention) and maintenance treatment (prophylactic treatment). Placebo-controlled maintenance treatment studies show prophylactic efficacy for imipramine (Frank et al, 1991), nortriptyline (Reynolds et al, 1999), fluoxetine (Montgomery et al, 1988), fluvoxamine (Terra \& Montgomery, 1998) and sertraline (chronic depression; Keller et al, 1998). The antidepressant efficacy of citalopram, a most effective selective serotonin reuptake inhibitor (SSRI), has been demonstrated in acute trials (see Noble $\&$ Benfield, 1997) and in two relapse prevention studies (Montgomery et al, 1993; Robert \& Montgomery, 1995). The present study investigates the prophylactic efficacy of citalopram in high-risk patients with recurrent major depression, using a threephase prophylaxis design.

\section{METHOD}

\section{Patient population}

Patients were in- and out-patients recruited from 54 centres (psychiatric institutions and, in the UK, general practitioner research settings) between November 1995 and January 1997 in nine European countries (Austria, Belgium, Finland, France, Italy, The Netherlands, Norway, Switzerland and the UK). They were of either gender, aged 18-65 years, suffering from unipolar recurrent major depressive episode (DSMIV, 296.3; American Psychiatric Association, 1994), had a total score $\geqslant 22$ on the Montgomery-Åsberg Depression Rating Scale (MADRS; Montgomery \& Åsberg, 1979) and had had two or more major depressive episodes prior to the index episode, the last one within the past 5 years.

Patients were excluded from the study if: the index episode had lasted more than 6 months; they had a history of schizophrenia, (hypo)mania, epilepsy, drug or alcohol misuse; a family history of bipolar disorder; or they suffered from severe somatic disorders. Similarly, patients were excluded if they had been treated recently with other antidepressants or electroconvulsive therapy ( 3 days to 8 weeks before entry, depending on treatment type), if they had a score $\geqslant 5$ for MADRS item 10 (suicidality) or if they were pregnant.

No concomitant psychotropic medication was allowed, except benzodiazepines and other hypnotics, the dose of which was to remain unchanged after week 8 of Period II and which could not be started in Periods II or III except in the case of relapse/recurrence.

All patients gave written informed consent prior to inclusion.

\section{Study design}

The study consisted of three treatment periods, outlined in Fig. 1: 6-9 weeks of open, acute treatment with citalopram (Period I); 16 weeks of open continuation treatment with citalopram to consolidate remission (Period II); and a minimum of 48 weeks of double-blind maintenance treatment with citalopram or placebo (Period III). The patients continued with double-blind treatment until the study ended in January 1998, resulting in individual treatment time spans of 48-77 weeks. The maximal total duration of treatment for completers was 102 weeks.

The initial dose of citalopram was $20 \mathrm{mg} /$ day. After 3 and/or 6 weeks, the dose was increased by $20 \mathrm{mg}$ if there was either an increase or no change in the Clinical Global Impression (CGI) severity of illness score (Guy, 1976) compared with the score 3 weeks earlier, or if the absolute score was $\geqslant 5$. At week 6, patients entered Period II if their MADRS score was $\leqslant 11$. Patients not meeting the entry criterion for Period II at week 6 continued in Period I until week 9. If they met the entry criteria at week 9 , they entered Period II; alternatively, they were 


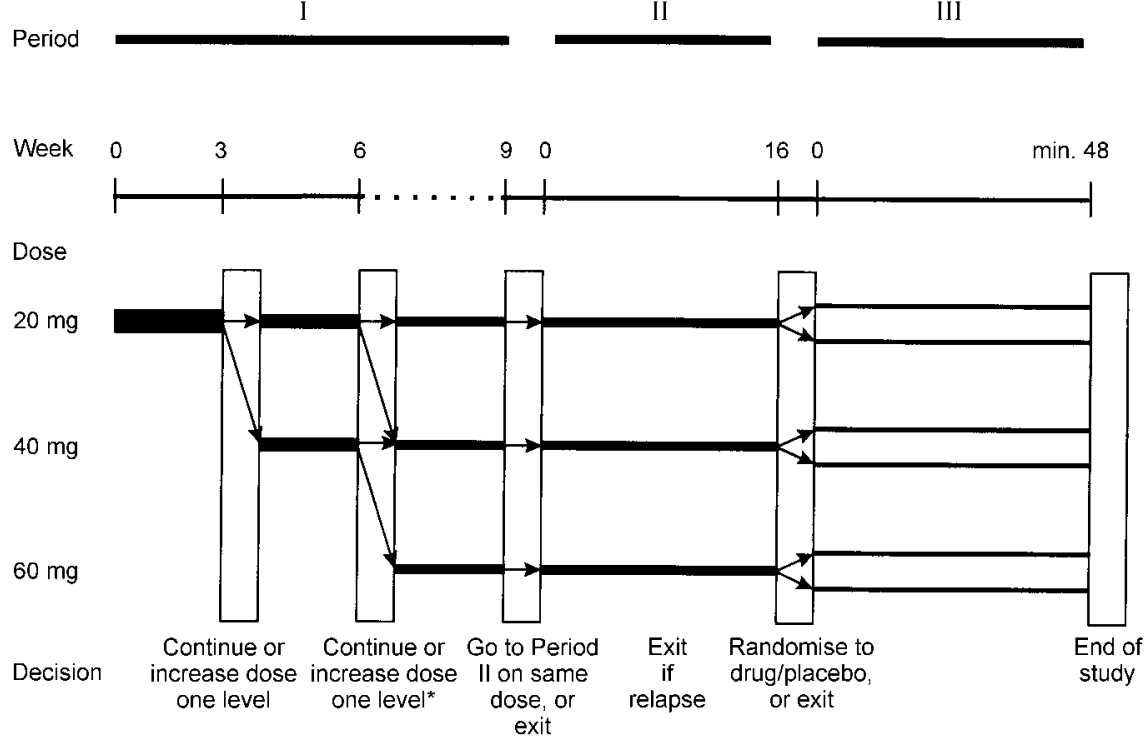

Fig. I Study design (see text for details). The asterisk indicates that eligible patients can enter Period II at week 6.

withdrawn. During Period II, the daily dose of citalopram remained fixed at that reached in Period I, i.e. 20, 40 or $60 \mathrm{mg}$. In Period II, patients were withdrawn if they experienced a relapse (MADRS $\geqslant 22$, confirmed after 3-7 days). All patients completing Period II with a MADRS score $\leqslant 11$ were randomised, using a block size of two (unknown to investigators), to receive double-blind treatment with either placebo or citalopram in Period III (same dose as in Period II). The randomisation was done on a 1:1 basis, irrespective of citalopram dose and without any stratification procedure.

Patients were seen every 3 weeks in Period I and monthly in Periods II and III, with one additional visit at week 2 of Period III. The CGI and MADRS ratings were performed at every visit, and Life Event Scale (American Psychiatric Association, 1987) ratings were performed at baseline, prior to randomisation, at week 24 of Period III and at the last visit. Concomitant medication and adverse events (assessed by open questions) were noted at each visit. Patient history and physical examination were registered at baseline. Laboratory tests were performed at baseline and prior to randomisation, and vital signs were assessed at these times as well as in Period III at week 24 and at the last visit. Withdrawn patients were treated at the investigator's discretion.

\section{Outcome measures and definitions}

The primary outcome measure was the time from randomisation (Period III) to recurrence of a new depressive episode.
Recurrence was defined as a MADRS total score $\geqslant 22$, confirmed after 3-7 days. An independent review board (three nonrecruiting-study advisory board members) evaluated the few ambiguous premature discontinuations prior to unblinding of the treatment code. Discontinuations judged by the review board as being recurrences were classified as such, even if assessed otherwise by the investigator.

Secondary outcome measures were MADRS and CGI score development. These data will be presented in a separate paper.

\section{Statistical analysis}

In order to have a power of at least $85 \%$ at a $5 \%$ significance level when using the log rank test for comparing time to recurrence, an estimated minimum sample size of 200 patients treated for a minimum of 48 weeks in Period III (100 per group) was calculated (see Goldman \& Hillman, 1992). This was estimated to require the inclusion of about 500 patients in Period I.

In the primary analysis, the log rank test was used to compare the time to recurrence for the citalopram and placebo groups. Overall treatment effect was estimated using the Cox proportional hazards model (Kragh Andersen, 1992). A set of potentially relevant covariates was selected (baseline characteristics, including age, age at onset, gender, number of previous episodes, family history of depression, citalopram dose and any kind of present and previous concomitant treatment) and evaluated on an exploratory level using the Cox proportional hazard regression model. First, all covariates were investigated in a model with treatment and the covariate. No interaction between treatment and covariates was found, thus interaction terms were not included. Based on these results it was decided to use forward selection to identify a set of covariates having a statistically significant effect on time to recurrence, starting with the model containing only treatment.

In the time-to-event analysis, patients were censored at discontinuation or major protocol deviations. Survival curves were estimated using the Kaplan-Meier method. The assumption of proportional intensities for the Cox proportional model with treatment effect was verified by plotting the estimated integrated intensity for the placebo group against the citalopram groups and by plots of the weighted Schoenfeld residuals (Therneau et al, 1990). The adequacy of using continuous covariates as linear predictors in the model was verified by plotting Martingale residuals against the covariate.

\section{RESULTS}

\section{Patients}

A total of 427 patients started the open 6-9-week acute treatment period (Period I), 327 of whom fulfilled the response criterion to enter the 16-week continuation treatment (Period II): 129 (39\%) on $20 \mathrm{mg}, 163$ (50\%) on $40 \mathrm{mg}$ and $35(11 \%)$ on $60 \mathrm{mg}$. The main reasons for discontinuation in Period I were lack of efficacy $(11 \%)$, adverse events $(5 \%)$, loss to follow-up ( $2 \%)$, withdrawal of consent $(2 \%)$ and non-compliance $(1 \%)$. In Period II, 58 patients discontinued, the main reasons being lack of efficacy $(5 \%)$, withdrawal of consent $(3 \%)$, administrative reasons $(3 \%)$, adverse events $(2 \%)$, loss to follow-up (2\%) and non-compliance (1\%). A total of 269 patients thus completed the continuation period and met the randomisation criteria to enter the double-blind prophylactic treatment (Period III) on either citalopram (132 patients continuing on $20 \mathrm{mg}$ (53), $40 \mathrm{mg}$ (66) or $60 \mathrm{mg}$ (13)) or placebo (137 patients who, prior to randomisation, had received $20 \mathrm{mg}$ (53), $40 \mathrm{mg}$ (74) or $60 \mathrm{mg}$ (10) of citalopram). Prior to unblinding of the treatment code, five patients were excluded from the intention-to-treat (ITT) population due to major protocol deviations; thus, the ITT 
population consisted of 264 patients (132 on citalopram; 132 on placebo).

The ITT population consisted of $29 \%$ males and $71 \%$ females, in good agreement with the known male:female ratio of lifetime prevalence of recurrent unipolar depression. The male:female ratio was slightly lower in the placebo group $(25 \%$ males) than in the citalopram group $33 \%$ males). The only statistically significant difference in baseline variables was seen for age of onset of first depression, which was slightly lower in the placebo group $(P=0.0115)$. The median number of previous depressive episodes (four in the citalopram group and three in the placebo group) and median time since first treatment started (8 and 6 years in the citalopram and placebo groups, respectively) were rather high and similar in both groups (Table 1). There were no differences in CGI score distribution: about one-third of patients in both groups were rated as moderately ill and the rest as markedly ill or above. Alcohol consumption was similar between treatment groups. Concurrent medical conditions were noted for $68.9 \%$ in the citalopram group and $62.1 \%$ in the placebo group. In both groups, the medical conditions most frequently reported were hypertension, menopausal/climacteric states, arthrosis, dorsalgia, asthma, various allergies, migraine and other headaches. Of the patients in both groups, $99.2 \%$ had previously received treatment for depression, with mainly good results. The frequencies of previous treatments were comparable between the two groups (antidepressant medication, 96.2\%; psychotherapy, 31.1$31.8 \%$; electroconvulsive therapy, $0.8 \%$ ).

\section{Acute and continuation treatment phases}

For the ITT population, the baseline tota MADRS score (s.d.) at entry into Period I was 30.4 (s.d.=4.97) in the citalopram group and $30.6(s . d .=4.78)$ in the placebo group, decreasing to 6.7 (s.d.=3.32) and 7.0 (s.d.=3.33), respectively, at the end of Period I. The corresponding initial MADRS scores for the different dose groups (citalopram/placebo) were as follows: $20 \mathrm{mg}$, $29.0 / 31.3$; $40 \mathrm{mg}, 31.0 / 30.3 ; 60 \mathrm{mg}, 33.4 /$ 29.4. The average entry score into Period III was $4.2(\mathrm{~s} . \mathrm{d} .=3.4)$ in the citalopram group and 4.3 (s.d.=3.4) in the placebo group.

\section{Prophylactic treatment phase}

For the placebo group of 132 patients 59 recurrences were observed in a total of
Table I Demographic and baseline characteristics of the intention-to-treat patient sample entering prophylactic treatment

\begin{tabular}{lcc}
\hline & $\begin{array}{c}\text { Period III: citalopram } \\
(n=I 32)\end{array}$ & $\begin{array}{c}\text { Period III: placebo } \\
(n=132)^{\prime}\end{array}$ \\
\hline Gender ratio (male/female) & $43 / 89$ & $33 / 99$ \\
Age (years) & $43.8 \pm 9.7$ & $42.4 \pm I I .5$ \\
Age at first depressive episode & $32.3 \pm 10$ & $30.6 \pm 12.2$ \\
MADRS at entry Period I & $30.4 \pm 5.0$ & $30.6 \pm 4.8$ \\
No. of previous episodes & $4(2-15)$ & $3(2-20)$ \\
No. of episodes during past 5 years & $2(1-10)$ & $2(1-10)$ \\
Time since first treatment start (years) & $8(0-37)$ & $6(0-35)$ \\
Time between last and present episode (months) & $12(2-60)$ & $12(1-58)$ \\
Duration of present episode (days) & $37(0-182)$ & $21(0-182)$ \\
Ever attempted suicide (yes (\%)/no (\%)) & $20(15.2) / 112(84.8)$ & $26(19.7) / 106(80.3)$ \\
Family history of depression (yes (\%)/no (\%)) & $38(28.8) / 94(71.2)$ & $52(39.4) / 80(60.6)$ \\
Hospitalisation (yes (\%)) & & \\
$\quad$ Previous & $25(18.9)$ & $27(20.5)$ \\
Present & $9(6.8)$ & $7(5.3)$ \\
\hline
\end{tabular}

I. Five patients randomised to placebo treatment were excluded from analysis prior to code break owing to major protocol deviations (see text for details).

MADRS, Montgomery-Åsberg Depression Rating Scale.
77.6 treatment years. For the citalopram group, consisting of 132 patients, 24 recurrences were observed in a total of 108.9 years (Table 2). The log rank test to compare the time to recurrence showed a statistically significant difference (log rank test, $\chi^{2}=24.4$, d.f. $\left.=1, P<0.001\right)$. From the Kaplan-Meier survival curves for citalopram and placebo groups in Period III (Fig. 2), it is seen that the time to recurrence is longer in the citalopram group than in the placebo group. Using a Cox regression model including treatment only, the risk ratio (ratio of the estimated hazard in the citalopram group over that in the placebo group) was estimated at $0.321 \quad(95 \%$ confidence interval $=0.199-0.516$ ).

Using forward selection when modelling the time to recurrence in a multivariate Cox proportional hazards model, treatment effect, age, MADRS score at entry into
Period I (both as linear and continuous predictors) and treatment by general practitioner (yes/no) (Table 3) were identified as covariates. mates of time to recurrence separately for each of the three dose levels. Although the study was not powered for subgroup analyses, the difference in time to recurrence between citalopram and placebo groups was statistically significant at all dose levels (log rank test: $20 \mathrm{mg}, P=0.0043 ; 40 \mathrm{mg}$, $P=0.0008 ; 60 \mathrm{mg}, P=0.0157$ ).

\section{Safety and tolerability}

In Period $\mathrm{I}$, the most frequent adverse events ( $\geqslant 5 \%$ in either treatment group) in the 427 patients treated with citalopram were nausea, headache, insomnia, somnolence, anxiety, influenza-like symptoms,
Figure 3 shows the Kaplan-Meier esti-

Table 2 Crude rate of recurrence during prophylactic treatment in citalopram and placebo groups in the intention-to-treat population

\begin{tabular}{lcccc}
\hline & $\begin{array}{c}\text { No. of } \\
\text { patients }\end{array}$ & $\begin{array}{c}\text { Person-years at } \\
\text { risk' }^{1}\end{array}$ & $\begin{array}{c}\text { No. of } \\
\text { recurrences }\end{array}$ & $\begin{array}{c}\text { Crude rate of } \\
\text { recurrence }^{2}\end{array}$ \\
\hline Citalopram & 132 & 108.9 & 24 & 0.22 \\
Placebo & 132 & 77.6 & 59 & 0.76 \\
\hline
\end{tabular}

I. Person-years at risk (PYR) is calculated as the number of patients multiplied by the number of years of exposure to treatment after randomisation.

2. The crude rate is calculated as the number of recurrences per PYR. Based on the PYR and crude rate, a rate ratio of $0.22 / 0.76=0.29(95 \% \mathrm{Cl} 0.18-0.47)$ may be calculated (see text for details). 


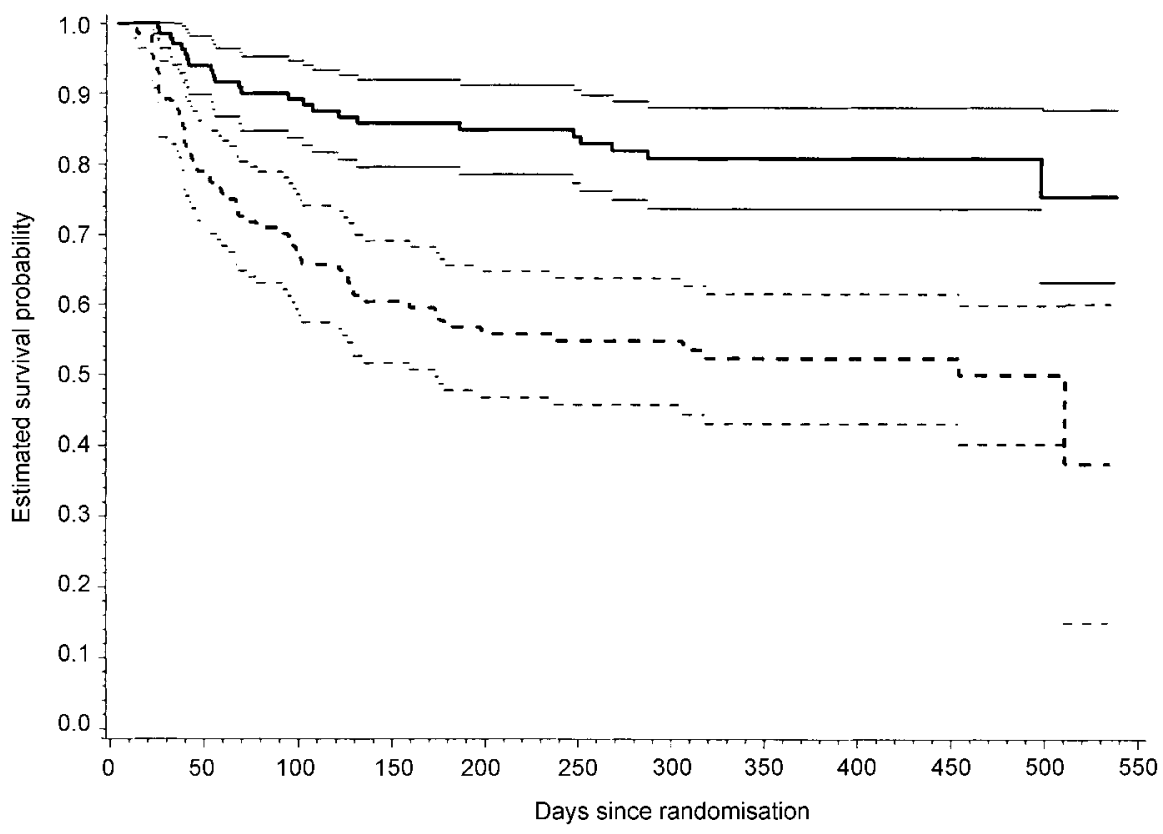

Fig. 2 Kaplan-Meier estimates of the time to recurrence of depression in the intention-to-treat population for citalopram group ( $n=132$, bold unbroken line) and placebo group ( $n=132$, bold dashed lines). The 95\% confidence intervals are shown as thinner lines. The difference in time to recurrence was statistically significant (log rank test, $P<0.0001$ ). The number of patients remaining in the study for 500 days or longer was 14 in the citalopram group and II in the placebo group.

Table 3 Risk ratios and $95 \%$ confidence intervals obtained using a multivariate Cox proportional hazards model'

\begin{tabular}{lccc}
\hline Parameter & Variable type & Estimate & 95\% confidence intervals \\
\hline Citalopram treatment & Categorical (yes/no) & 0.319 & $0.198-0.513$ \\
Age & Linear (unit I year) & 0.971 & $0.952-0.99 I$ \\
MADRS at entry into Period I & Linear (unit I point) & 1.057 & $1.014-1.102$ \\
Treatment by general practitioner & Categorical (yes/no) & 1.661 & $1.070-2.580$ \\
\hline
\end{tabular}

I. Forward selection was used to identify the clinically significant covariates that had a statistically significant effect on time to recurrence. The assumption of proportional intensities was verified by inspecting plots of the estimated integrated intensities. The adequacy of using continuous covariates (age and MADRS at entry into Period I) as linear predictors was verified using plots of the weighted Schoenfeld residuals against the covariate. No evidence was found for interaction between treatment and either of the covariates used in the model.

MADRS, Montgomery-Åsberg Depression Rating Scale.

dizziness and increased sweating. In Period III, the adverse event profile in the citalopram group was comparable to that of the placebo group, the most frequent events being (in order of descending frequency): in the citalopram group - headache, insomnia and back pain; in the placebo group dizziness, headache and insomnia (Table 4). If corrected for actual exposure, the frequency of adverse events in the citalopram group would have been reduced relative to that in the placebo group, given the considerably longer mean exposure in the former. Symptoms related to sexual function (ejaculation failure/anorgasmia, decreased libido) were reported with very low frequency $(<2.5 \%)$, and weight gain was not reported in the citalopram group at all (open questioning).

A few symptoms occurred at a higher frequency in the placebo group than in the active treatment group in Period III, notably dizziness, headache, nausea and vertigo in the first few weeks following randomisation (Table 5), suggesting the presence of potential discontinuation symptoms. The increase in intensity of these possible citalopram discontinuation symptoms appeared to be transient. Thus, for events starting in the placebo group on days $2-14$ after randomisation, the median duration of an episode was 21 days for dizziness, 19 days
Table 4 Frequency of Period III patients ( $n$, (\%)) with adverse events $(\geqslant 5 \%$ in either of the treatment groups)'

\begin{tabular}{|c|c|c|}
\hline & $\begin{array}{c}\text { Period III } \\
\text { Citalopram } \\
(n=132)\end{array}$ & $\begin{array}{c}\text { Period III } \\
\text { Placebo } \\
(n=137)\end{array}$ \\
\hline Headache & $22(16.7)$ & $21(15.3)$ \\
\hline Insomnia & 21 (15.9) & $20(14.6)$ \\
\hline Back pain & $18(13.6)$ & $20(14.6)$ \\
\hline Influenza-like symptoms & $18(13.6)$ & $15(10.9)$ \\
\hline Bronchitis & $15(11.4)$ & $9(6.6)$ \\
\hline Dizziness & II (8.3) & $22(16.1)$ \\
\hline Somnolence & II (8.3) & $10(7.3)$ \\
\hline Pain & $9(6.8)$ & $7(5.1)$ \\
\hline Abdominal pain & $8(6.1)$ & $13(9.5)$ \\
\hline Nausea & $8(6.1)$ & $14(10.2)$ \\
\hline Pharyngitis & $8(6.1)$ & $6(4.4)$ \\
\hline Sweating increased & $8(6.1)$ & $12(8.8)$ \\
\hline Arthralgia & $7(5.3)$ & $8(5.8)$ \\
\hline Chest pain & $7(5.3)$ & $4(2.9)$ \\
\hline Sinusitis & $7(5.3)$ & $5(3.6)$ \\
\hline Trauma, non-pathological & $7(5.3)$ & $10(7.3)$ \\
\hline Anxiety & $6(4.5)$ & $9(6.6)$ \\
\hline Fatigue & $6(4.5)$ & $5(3.6)$ \\
\hline Urinary tract infection & $6(4.5)$ & $3(2.2)$ \\
\hline Vomiting & $6(4.5)$ & $4(2.9)$ \\
\hline Accidental overdose & $5(3.8)$ & $2(1.5)$ \\
\hline Coughing & $5(3.8)$ & $3(2.2)$ \\
\hline Diarrhoea & $5(3.8)$ & $3(2.2)$ \\
\hline Rhinitis & $5(3.8)$ & $8(5.8)$ \\
\hline $\begin{array}{l}\text { Upper respiratory } \\
\text { tract infection }\end{array}$ & $5(3.8)$ & $10(7.3)$ \\
\hline
\end{tabular}

I. Events are sorted by the frequency in the citalopram treatment group.

for headache, 11 days for vertigo and 10 days for nausea.

The number of premature discontinuations owing to adverse events during prophylactic treatment was similar in the citalopram and placebo groups and there was no difference in the time to these discontinuations ( $\log$ rank test). One death, by suicide, occurred during the open treatment phase and only one serious adverse event (whiplash injury after car accident) that was rated by both sponsor and investigator as possibly related to treatment. There was one myocardial infarction, for which causal relation to treatment was assessed as possible by the investigator and unlikely by the cardiologist. Two manic reactions occurred, one (rated a serious adverse event) in Period I and the other in a 


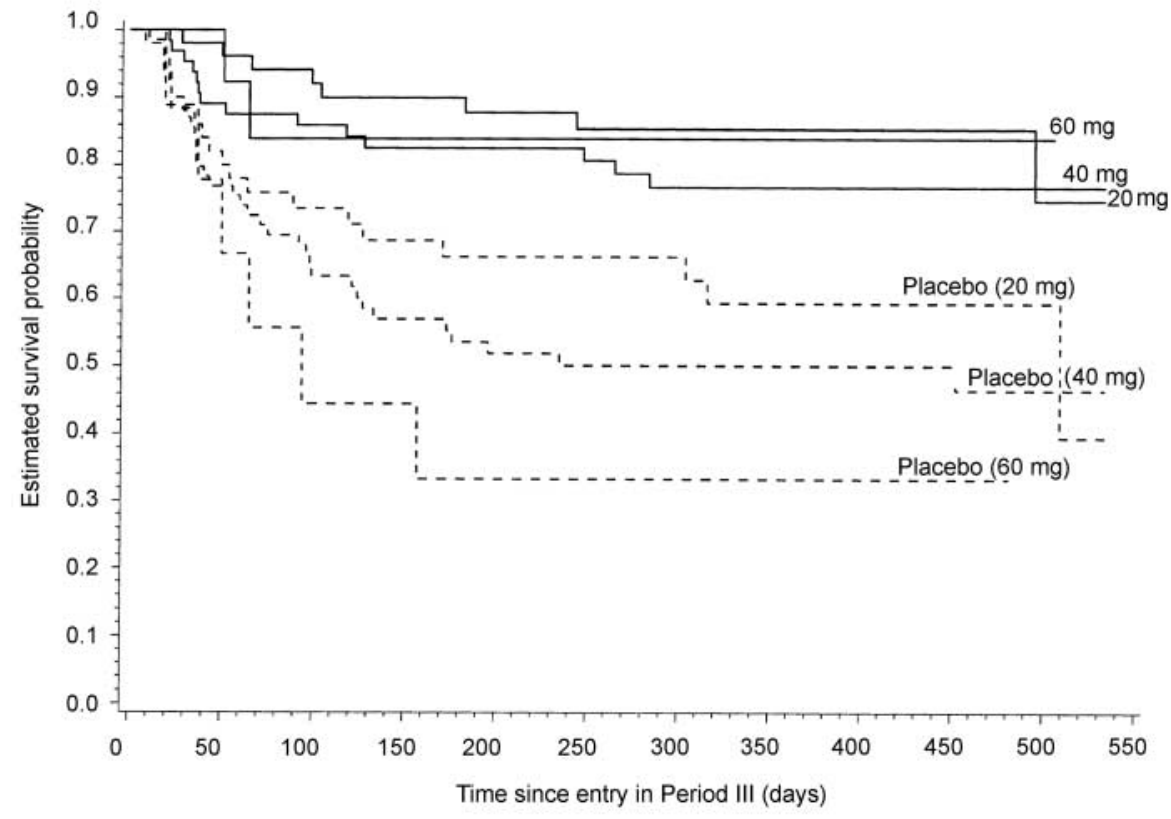

Fig. 3 Kaplan-Meier estimates of the time to recurrence in the intention-to-treat population, stratified by dose: citalopram group $(20 \mathrm{mg} /$ day, $n=53 ; 40 \mathrm{mg} /$ day, $n=66 ; 60 \mathrm{mg} /$ day, $n=13$ ) shown as unbroken lines; placebo group ( $20 \mathrm{mg} /$ day, $n=52 ; 40 \mathrm{mg} /$ day, $n=71 ; 60 \mathrm{mg} /$ day, $n=9$ ) shown as dashed lines. The dose for the placebo group was that received in Period II. The difference in time to recurrence was statistically significant at all dose levels (log rank test: $20 \mathrm{mg}, P=0.0043 ; 40 \mathrm{mg}, P=0.0008 ; 60 \mathrm{mg}, P=0.0157$ ).

Table 5 Number of patients with a change in adverse event profile during the first 2 weeks after randomisation'

\begin{tabular}{lccccc}
\hline & \multicolumn{2}{c}{ Citalopram } & & \multicolumn{2}{c}{ Placebo } \\
\cline { 2 - 3 } \cline { 5 - 6 } \cline { 5 - 6 } Diminished & Worsening & & Diminished & Worsening \\
\hline Dizziness & 0 & 1 & & 0 & 17 \\
Headache & 1 & 4 & & 0 & 12 \\
Nausea & 0 & 3 & & 1 & 8 \\
Vertigo & 0 & 0 & & 0 & 5 \\
\hline
\end{tabular}

I. Possible discontinuation symptoms were evaluated by assessing, for each patient and adverse event, whether the event was present at randomisation (day I of prophylactic treatment) and whether, within days 2-14, the event appeared, stopped or changed intensity with respect to status at day I.

patient taking placebo in Period III. Changes in laboratory or vital sign parameters were seen occasionally but were all essentially single-parameter changes unlikely to be related to the use of citalopram.

\section{DISCUSSION}

\section{Prophylactic efficacy}

The present findings demonstrate the prophylactic efficacy of citalopram in recurrent depression. This conclusion is based on the observed. The advantage of citalopram over placebo was significant at all three dose levels to which the individual patients responded during acute treatment of the index episode $(20,40$ or $60 \mathrm{mg}$ ).

\section{Study design}

Unlike many other studies of antidepressant efficacy in maintenance treatment, the present study employed a three-phase design with a placebo-controlled maintenance phase of sufficient duration, using a patient population at high risk of recurrence (Frank et al, 1991; Keller \& Boland, 1998). The acute treatment phase was designed to optimise response to citalopram (flexible dose and treatment extension up to 9 weeks), possibly explaining the low frequency of discontinuation owing to lack of efficacy. The patients had had, on average, 4.4 previous episodes and thus carried a high risk of recurrence. Several definitions for recurrence of depression have been used previously, ranging from reaching threshold value in a depression rating scale (Montgomery et al, 1988), to combinations with independent clinical judgement and confirmation of DSM criteria for depression (Frank et al, 1990; Keller et al, 1998). In the present study, recurrence of depression was defined as a MADRS total score of at least 22, confirmed after 3-7 days (upon randomisation, the average MADRS score was 4), and ambiguous cases were reviewed by an independent review board.

The results of studies of imipramine (Frank et al, 1990) and paroxetine (Franchini et al, 1998) have indicated that the full dose of antidepressants is more effective during prophylactic treatment than a reduced dose. Therefore, in the present study, the dose to which the patients responded during acute treatment and remained well on during continuation treatment was maintained during prophylactic treatment. Under these conditions, significant prophylactic efficacy of citalopram was found at doses of 20, 40 and $60 \mathrm{mg}$. For patients randomised to placebo, there was a trend towards increasing recurrence rates with increasing doses prior to randomisation. Interpretation of this finding should, however, be done with caution because of the unequal group sizes.

The design of the present study is similar to that used by Frank et al (1990) with respect to definitions of treatment phases 
and recurrence, optimisation of response and use of unchanged dose during maintenance treatment. In their study a 3-year maintenance period was used for all patients, whereas in the present study patients were followed for individual time spans, using survival analysis. From a crude comparison of the results it appears, however, that the prophylactic efficacy of citalopram is similar to that of imipramine in a highrisk population of patients suffering from recurrent depression. The present findings thus provide further support for the efficacy of SSRIs in prophylactic treatment of depression (Keller et al, 1998; Montgomery et al, 1988; Terra \& Montgomery, 1998) in a patient population responding to acute treatment with an SSRI.

\section{Potential contributing factors}

Analysis of the potential influence of clinically relevant covariates revealed that MADRS score at entry into Period I, age and treatment by a general practitioner significantly affected the risk of recurrence. For the MADRS score at entry as a continuous, linear predictor, a risk ratio of 1.057 was found, suggesting that the risk of recurrence increases with increasing severity of illness. In apparent agreement, the baseline CGI score was the only significant predictor of time to recurrence in a study of the prophylactic efficacy of sertraline (Keller et al, 1998). A risk ratio of 0.971 was found for age as a continuous predictor. The risk of recurrence was greater in younger patients in both treatment groups, but the effect of age was less in the citalopram group, indicating that younger patients may benefit most from prophylactic treatment. The decreased risk of recurrence with increasing age is in contrast to other published findings (Angst, 1988). Possibly, the present result may be affected by other covariates, such as earlier age of onset in the young patients, which is a known risk factor (Angst, 1988). Moreover, complex effects of age at first admission have been reported, suggesting that after more than one episode the risk of recurrence may decrease with older age (Kessing, 1998; Kessing \& Andersen, 1999).

A risk ratio of 1.661 was found for treatment by general practitioner as a dichotomous variable, reflecting a relatively higher recurrence rate in the patients in this setting. The reasons for this phenomenon remain to be elucidated. Tentatively, it may be suggested that the general practitioners may recruit a patient population, characterised by a different medical and psychiatric comorbidity profile than patients recruited by psychiatric institutions. Moreover, observation bias cannot be excluded, because the evaluators in the general practitioner settings were specially trained, nontreating nurses, whereas in other centres they were the treating physicians.

The number of previous episodes was not a predictor of recurrence in the present study, in contrast to previous reports (NIMH/NIH Consensus Development Panel, 1985; Angst, 1988). This might reflect a possible ceiling effect, given the high mean number of previous episodes in the patient sample, and could also reflect confounding by age (given the above suggested relationship between age and number of previous episodes as predictors).

In contrast to previous findings that family history may be a predictor of course and outcome (Duggan et al, 1998), we found no association between a positive family history of depression and risk for recurrence. This may be due to differences in the methodology of patient sampling and the definition of family history and outcome.

\section{Tolerability}

Citalopram was well tolerated during long-term treatment; adverse events were generally mild or moderate in intensity. Weight gain was not reported in citalopram patients at all, and sexual symptoms were reported with very low frequency. An increase in the frequency of certain adverse events, particularly dizziness, headache and nausea, was observed in the placebo group shortly after randomisation. The symptoms were transient (median duration maximally 3 weeks) and were mild or moderate in intensity. Based on the findings in the present study, it appears that acute discontinuation of citalopram may be associated with mild discontinuation symptoms. Similar symptoms have been found in relation to discontinuation of other SSRIs, the most commonly reported being influenza-like symptoms, along with memory impairment, insomnia, shock sensation, headache, anxiety and agitation/aggression (Rosenbaum et al, 1998). Finally, it may be noted that many questions regarding long-term prophylactic treatment of patients with recurrent depression still remain, including the optimal duration and long-term effects of prophylactic treatment, and the influence of clinical predictors on outcome.

\section{ACKNOWLEDGEMENTS}

The authors gratefully acknowledge all individual investigators participating in the study for their excellent work, and Professor S. A. Montgomery for careful review of the study protocol and rating training of investigators.

\section{REFERENCES}

American Psychiatric Association (1987) Diagnostic and Statistical Manual of Mental Disorders (3rd edn, revised) (DSM-III-R), p. II.Washington, DC: APA

- (1994) Diagnostic and Statistical Manual of Menta Disorders (4th edn) (DSM-IV). Washington, DC: APA

Angst, J. (1980) Verlauf unipolar depressiver, bipola manisch-depressiver und schizoaffektiver Erkrankungen und Psychosen. Ergebnisse einer prospektiven Studie. Fortschritte der Neurologie-Psychiatrie und Iher Grenzgebiete, 48, 3-30.

_ (1988) Risikofaktoren für den Verlauf affektiver Störungen. In Affektive Störungen, diagnostische, epidemiologische, biologische und therapeutische Aspekte (eds D. v. Zerseen \& H. J. Möller), pp. 99-II0. Springer Verlag: Berlin.

Duggan, C., Sham, P., Minne, C., et al (1998) Family history as a predictor of poor long-term outcome in depression. British Journal of Psychiatry, 173, 527-530.

Franchini, L., Gasperini, M., Perez, J., et al (1998) Dose-response efficacy of paroxetine in preventing depressive recurrences: a randomised, double-blind study. Journal of Clinical Psychiatry, 59, 229-232.

Frank, E., Kupfer, D. J., Perel, J. M., et al (1990) Three-year outcomes for maintenance therapies in recurrent depression. Archives of General Psychiatry, 47, 1093-1099.

, Prien, R. F., Jarret, R. B., et al (1991)

Conceptualisation and rationale for consensus definitions of terms in major depressive disorder. Archives of General Psychiatry, 48, 85I-855.

_ , Kupfer, D. J., Perel, J. M., et al (1993) Comparison of full-dose versus half-dose pharmacotherapy in the maintenance treatment of recurrent depression. Journal of Affective Disorders, 27, 139-145.

Goldman, A. L. \& Hillman, D. W. (1992) Exemplary data: sample size and power in the design of event-time clinical trials. Control Clinical Trials, 3, 256-27l.

Guy, w. (1976) ECDEU Assessment Manual for Psychopharmacology. Revised, DHEW Publication (ADM) No. 76-338. Rockville, MD: National Institute for Mental Health.

Keller, M. B. \& Boland, R. J. (1998) Implications of failing to achieve successful long-term maintenance treatment of recurrent unipolar major depression. Biological Psychiatry, 44, 348-360.

_ , Kocsis, J. H., Thase, M. E., et al (1998)

Maintenance phase efficacy of sertraline for chronic depression. Journal of the American Medical Association, 280, 1665-1672.

Kessing, L.V. (1998) Recurrence in affective disorder. II. Effect of age and gender. British Journal of Psychiatry, $\mathbf{1 7 2}$, 29-34. 
_ \& Andersen, P. K. (1999) The effect of episodes on recurrence in affective disorder: a case register study. Journal of Affective Disorders, 53, 225-231.

Kessler, R. C., McGonagle, K. A., Zhao, S., et al (1994) Lifetime and I2-month prevalence of DSM-III-R psychiatric disorders in the United States. Results from the National Comorbidity Survey. Archives of General Psychiatry, 51, 8-19.

Kragh Andersen, P. (1992) Statistical Models based on Counting Processes. Berlin: Springer.

Montgomery, S. A. \& Åsberg, M. (1979) A new depression scale designed to be sensitive to change. British Journal of Psychiatry, I34, 382-389.

_ , Dufour, H., Brion, S., et al (1988) The prophylactic efficacy of fluoxetine in unipolar depression. British Journal of Psychiatry, 153 (suppl. 3), 69-76.

_, Rasmussen, J. G. C. \& Tanghøj, P. (1993) A 24-week study of $20 \mathrm{mg}$ citalopram, $40 \mathrm{mg}$ citalopram, and placebo in the prevention of relapse of major depression. International Clinical Psychopharmacology, 8 , $181-188$

Mueller, T. I., Leon, A. C., Keller, M. B., et al (1999) Recurrence after recovery from major depressive disorder during 15 years of observational follow-up American Journal of Psychiatry, I56, 1000-1006.

\section{NIMH/NIH Consensus Development Panel (1985)} Mood disorders: pharmacologic prevention of recurrences. American Journal of Psychiatry, 12, 469-476.

Noble, S. \& Benfield, P. (1997) Citalopram. A review of its pharmacology, clinical efficacy, and tolerability in the treatment of depression. CNS Drugs, 8, 410-431.

Reynolds III, C. F., Frank, E., Perel, J. M., et al (1999) Nortriptyline and interpersonal psychotherapy as maintenance therapies for recurrent major depression: a randomized controlled trial in patients older than 59 years. Journal of the American Medical Association, 28I, 39-45.

Robert, P. \& Montgomery, S. A. (1995) Citalopram in doses of 20-60 mg is effective in depression relapse prevention: a placebo-controlled 6 month study. International Clinical Psychopharmacology, 10 (suppl. I), 29-35.

Rosenbaum, J. F., Fava, M., Hoog, S. L., et al (1998) Selective serotonin reuptake inhibitor discontinuation

\section{CLINICAL IMPLICATIONS}

Maintenance treatment with citalopram effectively reduces recurrent depressive episodes.

- Prophylactic efficacy was shown separately for daily dose levels of 20,40 and $60 \mathrm{mg}$ of citalopram.

Long-term tolerability and safety did not differ between citalopram and placebo.

\section{LIMITATIONS}

- The lengths of prophylactic maintenance treatment were between $\mathbf{4 8}$ and 77 weeks.

The study does not permit an evaluation of reducing dose levels during maintenance treatment.

- The age of the patient population was between 18 and 65 years.

B. HOCHSTRASSER, PhD, Privatklinik, Meiringen, Switzerland; P. M. ISAKSEN, MD, Haukeland sykehus, Psykiatrisk klinikk, Bergen, Norway; H. KOPONEN, PhD, Department of Geropsychiatry, Moisio Hospital, Mikkeli, Finland; L. LAURITZEN, MD, Hillerød Hospital, Psykiatrisk afd. P, Hillerød, Denmark; F. A. MAHNERT, MD, Universitätsklinik für Psychiatrie, Graz, Austria; F. ROUILLON, MD, Service de Psychiatrie Hôpital Albert Chenevier, Creteil, France; A. G.WADE, MD, Community Pharmacology Services Ltd, Glasgow, UK; M. ANDERSEN, MSc, S. F. PEDERSEN, MSc, J.C. G. DE SWART, MSc, R. NIL, PhD, International Clinical Research, H. Lundbeck A/S, Valby, Denmark

Correspondence: B. Hochstrasser, Privatklinik, CH-3860 Meiringen, Switzerland

(First received I2 January 2000, final revision 12 October 2000, accepted 13 October 2000)

syndrome: a randomized clinical trial. Biological Psychiatry, 44, 75-76.

Terra, J. L. \& Montgomery, S. A. (1998) Fluvoxamine prevents recurrence of depression: results of a long- term double-blind, placebo-controlled study. International Clinical Psychopharmacology, 13, 55-62.

Therneau, T. M., Grambsch, P. \& Fleming, T. (1990) Martingale-based residuals for survival models. Biometrika, 77, 147-160. 